\title{
The Need to "Rediscover" the Right in the I-the Other Dialectics
}

\author{
Arjan Vasjari PhD \\ Juelda Lamçe PhD
}

Doi:10.5901/ajis.2013.v2n2p155

European University of Tirana

\begin{abstract}
Which is the proper proprium of the right, in other words its essence? Is it possible to speak of a "single essence" of it? In our epoch is it sufficient the study of the right in its ontic dimension, as a fact, or it needs to be ontologically rediscovered, as a meaning/sense? In order to give an answer (a in-exhaustive one) to these questions posed to the contemporary right, one of the most reasonable ways is its rediscovering by means of a rational key, a rediscovery in the "I-the" other dialectics. It is not an unknown reading key. On the contrary, it is widely recognised, but paradoxically it is left aside: it has to do with the Aristotelian formula "the right belongs to the human as such, thus as a man". In other words, the right prior to being a rule and a norm needs to be conceptualised as e relationship. This means that, as an associative form the right needs to place at its hub the socius exigency, its social mutation, its capacity and need to adapt to the dynamics of the society where he lives. It cannot be but in doubt that the right remains a regulated form, but in the rule-relationship dichotomy it is (and it should always be) the rule in function to the relationship and never the reverse. If it was not this way then the right would be transformed into an arbitrary mechanism, without its basic cell, in other words without its recta ratio. Acknowledging the axiom that the rule is born to serve the rational relationship, we have directly acknowledged that it also needs to adapt itself to the dynamics of the relationship, thus with the social body dynamics. It is precisely for this reason that the contemporary right (in the former communist countries in particular) is obliged to face phenomena and problems that it simply needs to discipline and juridically regulate, but moreover it needs to give them a meaning. It needs to be accepted that the set of topics of the debate concerning the right has been broadened so much that it could not be imagined a few decades ago. This set of topics exceeds the matrix debate, the so-called traditional, including aspects of the philosophy of moral, legal anthropology, sociology, politics, and bioethics, etc. It is a confrontation that these disciplines need to make with the right, rediscovering this way a number of dimensions of the latter which have been eclipsed due to its limited normative dimension. Multiculturalism, legal plurality, and bio-juridical are some of these phenomena that these lines seek to address. Their particularity compared to the right, their imposition for a reconceptualisation of the right, the acceptance free of complexes of the limitations of the right.
\end{abstract}

Keywords: the right as a relationship, socius, coexistential experience, multiculturalism, legal pluralism, bio-juridical

\section{Premessa}

La crisi degli anni settanta del secolo scorso che colpì lo iuspositivismo, specialmente quello di orientamento hartiano, segna forse, una tra le trasformazioni più importanti del concetto "diritto" nell'epoca moderna. Gli anni novanta, e in particolar modo i cambiamenti politico-ideologici che hanno attraversato e cambiato la mappa del mondo, hanno accelerato questo cambiamento configurando così il diritto come il linguaggio della relazione sociale ma, al di fuori della cornice nazionale e monoculturale. La dicotomia classica "essere" e "deve essere" diventa non semplicemente insufficente ma superata. II diritto inizia e riconsiderare i suoi raporti con la morale, abbandonando cosi l'autosufficienza e la supremazia che lo aveva caratterizzato fino a quel momento. Dunque, il diritto cerca una sua morale, tant'è vero questo che, Lon Fuller parla nell'sua opera The morality of law, della "moralita interna della legge", moralita identificata da lui nei principi di rule of law.

D'altronde, i processi di globalizzazione, progresso scentifico l'abbraciarsi del multiculturalismo, tipici fenomeni del periodo post-moderno, impongono al diritto di rivedere se stesso. Questo non vuol dire che iusnaturalismo, iusrealismo e iuspositivismo spariscono. No! Vuol dire che molti teorici del diritto guardano oltre. L'allargamento tematico imprevisto, che include non soltanto questioni del dibatito tradizionale ma anche questioni della filosofia morale, sociologia, politica ecc. Impongono al diritto di confrontarsi e scoprire dimensioni, non identificate da essa fino ad allora. II diritto si diversifica sia dentro la comunità politica, sia a livello sovranazionale e internazionale. I scenziati del diritto iniziano di rielaborare il concetto regola e addirittura cominciano a riconcepire il diritto stesso, non soltanto come una scienza pura 
di norme ma, anche come una disciplina che ha bisogno di scoprire e adottare il ragionamento morale, il sistema del valori, le tecniche della democrazia, le mutazioni sociali, il pluralismo culturale e tutto quello che ha a che fare con l'anatomia in cambiamento della societas. II diritto non puo essere ridotto soltanto nell'interdipendenza kelseniana "violazione-sanzione" (quanto piu grande è la violazione, tanto piu grande sarà la sanzione). Dentro la sua esistenza, il diritto inizia a cercare l'equilibrio compromesso tra l'istituto del fatto e il valore etico-politico. Quindi, con una dose ragionevole di speculazione, possiamo dire: il diritto (iuspositivismo) contemporaneo è diventato meno "puro", e piu "ibrido"; esiste non solo in un unica dimensione ma in vari dimensioni; è costretto a confrontarsi non soltanto con altre discipline ma sopratutto si deve confrontare con se stesso. In altre parole, il diritto va riconcepito, risistemato, riadatatto e perchè no, rielaborato entro alcuni nuovi orizonti. Ma quali sono questi (alcuni) orizonti?

\section{II multiculturalismo}

II multiculturalismo sarebbe uno tra questi. Dobbiamo partire da alcune domande per capirlo. Può essere univoco il concetto "la verita della giustizia"? Se questa verità (secondo l'opinione comune) dovrebbe essere una e una solo, allora perchè le società, nel rispetto della loro cultura (giuridica) possiedono un loro concetto sulla verità della giustizia? Concetto il quale, entro il contesto ed il loro modo di concepire la giustizia, è giusto. La risposta contiene in sé il pericolo di un relativismo preso sul serio. Ma, se vogliamo realmente una risposta (senza pretenderla come esauriente), la possiamo trovare nel pluralismo culturale.

Ci sono due modi per intendere il multiculturalismo come teoria. II primo: "multiculturalismo definisce l'esistenza dentro il corpo sociale delle microcomunità con profili culturali (forse anche antropologici) diversi, caratterizzati da lingua, usi e costumi, ethos, modi di pensare differenti. II secondo,il multiculturalismo si puo spiegare in una visione giuridiconormativo che sottointende la tendenza di costruire un modelo giuridico-politico, possibile attraverso il coinvolgimento dei strumenti statali come il diritto e le istituzioni" (Faralli Carla, Filosofia del diritto contemporanea, Laterza, 2002).

Nella sociologia moderna del diritto non ci sono più dubbi circa il fatto che il socius nelle società moderne ha un'appartenenza plurima. Questo vuol dire fondamentalmente due cose: 1-che esso ha un identità frammentata, quindi non è monoidentitario; 2-esso è soggetto di modelli e norme le quali, non raramente, possono essere 0 in dissacordo, 0 in conflitto tra loro. Quindi, essendo di appartenenza plurima, il socius moderno condensa in sé un'appartenenza normativa altrettanto plurima. Esso dovrebbe riferirsi a una pluralità di norme che sono eterogenee e di diversi fonti, norme che si trovano non soltanto nei sistemi giuridici da dove vengono e, in particolarmente, dove vanno, ma anche a norme di natura religiosa, etnica, morale, sovranazionale ecc., spesso di validità personale 0 etnica e non territoriali. Quindi, sono norme le quali non neccessariamente sono riconosciute da sistemi statali ma, comunque, effetive. La regolarizzazione di una buona parte dei rapporti nel seno della comunità si realizza in maniera autonoma ma, anche se di importanza giuridica per le relazioni dentro il gruppo, questa regolarizzazione rimane al di fuori del controllo del sistema giuridico-istituzionale dello Stato ospitante. Tutta questa situazione, alla fine, è un puro prodotto del fenomeno di multiculturalismo. Oggi è un fatto innegabile che, il multiculturalismo è diventato un fenomeno universale e la consapevoleza della convivenza in armonia con esso,a Imeno nella civiltà occidentale, ha fatto sì che il monocentrismo culturale insieme al etnocentrismo, vengono vissuti come fenomeni superati e dall'altra parte si accetta come qualcosa di molto normale il politeismo delle culture. II riconoscimento e la protezione della tradizione culturale dei grupi in ambienti plurali è inseparabile dal riconoscimento e la protezione della libertà e l'identità, in tutte le sue variabili possibili. È la questione dei diritti dell'uomo, non la semplice proclamazione ma l'attuazione concretta, la quale fa scoprire il multiculturalismo come componente essenziale con il quale il diritto si deve adattare. II passaggio della protezione dei diritti dalla dimensione nazionale in quella regionale ha imposto in modo chiaro l'acettazione, non soltanto dei diversi sistemi giuridico-statali della protezione, ma anche una specializzazione relativa ad alcune tematiche particolari dei diritti dell'uomo. La costruzione dei sistemi regionali della difesa di questi diritti, raccoglie, prima di tutto spazi culturalmente diversi. Questo significa neccessità di unificare il concetto "diritti" in questi macrospazzi unificando in questo modo anche gli strumenti per la loro difesa. Quindi si va verso una concezione multiculturale dei diritti dell'uomo superando allo stesso tempo anche l'idea di stato-nazione per la loro protezione. La Convenzione Americana per i Diritti dell'Uomo, La Carta Europea dei Diritti dell'Uomo, La Carta Africana dei Diritti dell'Uomo, La Carta Araba dei Diritti dell'Uomo, sono documenti che hanno superato anche le differenze culturali degli Stati firmatari transformandosi in questo modo in strumenti di unificazione culturale di questi diritti. Ma non solo. La cosa piu importante è che questi documenti sono stati accolti ed accettati come strumenti giuridici omologati per la difesa di questi diritti.

L'altro aspetto, forse più problematico, che obbliga il diritto di prendere seriamente in considerazione l'eterogenita 
culturale, riguarda i flussi migratori e le trasformazioni demografiche che l'Occidente in particolar modo, sta affrontando. Quindi va detto: oggi, multiculturali, non sono soltanto le società. Multiculturale oggi è anche l'individuo.

Però, gli spostamenti di massa delle differenti culture verso spazi culturalmente altrettanto differenti, creano molti problemi relativi al bisogno di positivizzare usi e costumi i quali, puo succedere, di non essere conformi con gli usi e costumi della realtà sociale ospitante. Si implicano diritti, comportamenti, usi di natura religiosa, culturale, etico-morali ecc., i quali, spesso, non sono conformi con quelli della società ospitante, cosa che obbliga una soluzione di natura giuridica. In questo modo, il legislatore si trova di fronte alla neccesistà di normativizzare istituti specifici per grupi specifici, ammettendo in questo modo il fatto che, il pluralismo culturale può influenzare direttamente le politiche legjislative di una comunità plurale. Basta ricordare qui i concetti, culturalmenete differenti,d ei diritti della donna in Occidente e nel mondo arabo. Non è esagerato confermare che, l'impegno di risolvere queste discordanze concettuali attraverso strumenti di cooperazione intrareligioso, non può sostituire in nessun modo, il diritto. Non è lo sfondo culturale, o religioso, o morale, o sociale che va correto. Questo non è possibile. Anzi è utopico. Non c'è dubbio che, un soggetto si sarebbe trovato in una posizione marginale dentro una società dominata da un'altra cultura. Dal punto di vista culturale, questo incide non soltanto nella sua esperienza interrelazionale con l'altro e la società dove si trova ma, altrettanto inciderà, nella sua esistenza giuridica.

II ragionamento è semplice: sicuramente il soggetto si relazionerà giuridicamente. Però a causa della marginalità culturale (inclusa quella religiosa, etica, sociale ecc.) nella costruzione del rapporto giuridico egli non si relazionerebbe con I' altro semplicemente come socius, membro di communitas, come essere neutrale con il quale lo unisce l' obbiettività di una legge comune. La relazione giuridica ne risentirebbe (anche in silenzio) della sua posizione, culturalmente marginale, faccendo cosi che quell' alterum con il quale è costretto a relazionarsi, si percepirebbe non come deus ma come lupus. Ma, sopratutto, il sistema socio-giuridico della communità dove si trova non verrebbe accettato coscientemente come giusto ma soltanto come un'imposizione al quale non si pottrebbe fare altro che sottometersi. Questo farebbe in modo che egli vivesse questa societas come aliena, rifiutando in silenzio l' integrazione in essa e allo stesso tempo, non accettasse razionalmente, come rappresentante dei suoi interessi, il sistema dove si trova. Cioè, affinchè questo socius non si senta marginale a causa della sua cultura, allora è necessario che intervenga il diritto. Solo esso lo può liberare da quello status di esclusione; solo il diritto può risvegliare, in una dimensione ormai nuova, il senso di appartenenza; solo il diritto può offire garanzie alla non marginalizzazione. II diritto può rendere possibile tutto questo, attraverso la conoscenza, la positivizzazione e la difesa non semplicemente dei diritti universalmente riconosciuti ma, in particolare attraverso la positivizzazione anche dgli usi, comportamenti e codice morale della sua appartenenza (ovviamente non si parla di un diritto $a d$ hoc). Perchè è questa triade tramite la quale, quel socius, puo esprimere, più di ogni altra cosa, la sua esistenza identitaria. Ed è esattamente la positivizzazione di questa triade, la sua trasformazione in norma giuridica, che farebbe sentire a questo socius la sua identità e cultura di provenienza come parte integrale della cultura ed dell'identità della societas dove si è stabilito. Non per nulla i latini dicevano ubi societas, ibi ius!

\section{II pluralismo giuridico}

Anche se l'inizio dei dibatiti teorici sul pluralismo giuridico è legato con la sceinza giuridica americana (legal pluralism), è un fatto che, a sua elaborazione è stata avanzata in modo più articolato dopo le dinamiche della globalizzazione economica del secolo scorso. E' il periodo quando il concetto pluralismo giuridico inizia a creare confidenza nel circoli giuridici ma, allo stesso tempo,mette in crisi lo stesso concetto monista del diritto.

In linea generale, in una macrovisione, al concetto pluralismo giuridico ci si può approcciare in due modi, oggetivo e soggetivo. Secondo il primo approccio, il plurigiuridico definisce la coabitazione di diversi sistemi normativi i quali sono in una relazione comunicativa fra di loro, anche se autonomi rispettivamente. Secondo l'approccio soggetivo, il pluralismo giuridico rappresenta una situazione nel quale l'individuo è soggetto di più sistemi normativi allo stesso tempo. Va sottolineato, quello che diceva Gurvitch secondo il quale, il principio del monismo giuridico non è stato caratterizante per tutte le società. "Se il bonapartismo e la monarchia hanno costruito un'invenzione unitaria attraverso la razionalizzazione e la codificazione, l'esperienza del medioevo dominata dal pluralismo rappresenta una chiara prova come questa invenzione non appartiene a tutte le società. Di conseguenza, nel XX secolo, l'osservazione dei fenomeni giuridici va orientato nella riscoperta del pluralismo giuridico". (Gurvitch.G, La declaration des droits sociaux, Edizioni di comunita, Milano, 1949).

Non è difficile capire che, per un paradigma largamente condivisa che noi abbiamo circa il diritto, il pluralismo 
giuridico nella sua essenza è "antistatale", nel senso che tende di spostare il diritto al di fuori dal perimetro limitato dello Stato. Secondo questa teoria, il diritto statale non è l'unico diritto perchè: 1-esistono altrettanti diritti fuori ed indipendentemente dallo stato; 2-la legge dello Stato non è l' unica fonte di diritto ma uno tra questi. Proprio questo ci interesa capire ed accetttare allo stesso tempo, cioè di capire ed accettare che il diritto, ontologicamente, è una strutura universale che non accetta limiti. II concetto pluralismo giuridico ci interessa vederlo nel periodo post-moderno anche in un campo globale.

II succo è questo: il plurarlismo giuridico costituisce uno strumento attraverso il quale la scienza giuridica può incarnare quelle espressioni normative che vengono prodotte al di fuori dell'orbita limitata dello Stato. Ma, non solo questo: esso può diventare sostituto dei bisogni dei sistemi giuridici statali per trovare i molti punit in comune. Ed ancora, il pluralismo giuridico, non è effetto della globalizazzione economica ma ha lo status di compagno in questo viaggio a senso unico ormai dell'intera umanità. Ci basta constatare che nell' ra globale, i sistemi giuridici nazionali si confrontano quasi con lo stesso tipo di problemi. Il soggetto giuridico sempre più spesso si sta trasformando dall'altro esotico all'altro interno considerato ancheil fatto che il concetto interno sempre più spesso fuoriesce dalla sfera nazionale stabilizzandosi così in un campo globale .E veramente assiomaticoil fatto che oggi non esiste piu un'unica risposta giusta per lo stesso casus giuridico. Le risposte sono tante quanto lo sono i contesti storici, normativi, culutrali, morali, di valore ecc. Ma è altrettanto vero che proprio per l'affermazione di prima cioè che, il diritto è una dimensione universale, esso diventa sempre più spesso interrelazionale,la pratica giuridica diventa sempre più spesso sovranazionale e transnazionale, l'opinione giuridica si transforma in una ricchezza globale che appartiene a tutti, che serve a tutti e può essere un sistema di riferimento per tutti nonostante la stretta appartenenza nazionale degli utenti. L'avvicinamento, l'armonizazzione, l'integrazione e infine l'uniformizazzione del diritto tra gli Stati nello spazio comunitario ne è una prova evidente. Però la dimensione pluralizzante va al di là di questo perchè sempre più spesso si parla della circolazione degli istituti giuridici tra i diversi sistemi, si parla di un bisogno di divisione di questi isituti, cioè si parla di una globalizazzione giuridica, di una "universalizazzione del diritto positivo". (Cassese.S, Universalita del diritto, Napoli, Ed. Scientifica, pg.7,2005). Non è una coincidenza che per molti studiosi del diritto le referenza giuridiche devono essere allargate oltre i confini nazionali e che " non possono non essere tenuti in considerazione le soluzioni dei vari conflitti giuridici nel resto del mondo" (Waldron.J, Foreign law and the modern ius gentium, Harward Law Review, pg. 130, 2005). Lo stesso ragionamento vale anche per l'articolazione sempre più frequente nei circoli giuridici del concetto "giudizio equivalente", il quale sottointende da una parte l'abbandono del concetto monista del diritto cioè l'accetazione del formato pluarale della giurisprudenza anche dall'altra parte, la metodica interrelazionale entro essa. (Viola.F, Concorrenza degli ordinamenti e il diritto come scelta, Ed. Scentifica, Napoli, pg.65, 2008).

Sicuramente il pluralismo giurdico si confronta con molti rebus questi tempi. Rebus che includono prima di tutto aspetti isituzionali e più in là concetti per il diritto e la giustizia in se. Il bisogno sempre più forte per la comunicazione tra i sistemi giuridici a livello transnazionale, chiede senza dubbio una riformulazione del concetto di diritto, ma anche della sua applicazione in realtà tradizionalmente diverse, per scopi diversi. Perchè il diritto è assolutamente universale ma non deve essere negato che è anche contestuale. Ci sono esmpi che illustrano i rebus di cui abbiamo appena parlato. Secondo l'OBT "Government procurement agreement", impone che nella scelta dei contraenti, rispettando il principio di uguaglianza, le gare (appalti) devono essere aperti per tutti. Quindi, trasparenza, concorrenza, uguaglianza, scelta motivata. Ma possono essere applicati questi principi anche in realtà dove i contratti pubblici vengono stilati con l'obbiettivo di favorizzare zone meno sviluppate economicamente (zone dove vivono minoranze etniche per esempio)? Quindi, possono coesistere norme per il commercio internazionale fissate dal OBT con il bisogno di favorizzare categorie sociali prescelte?

Un simile rebus nasce anche per quanto riguarda l'istituto della consultazione, un diritto che viene riconosciuto al soggetto ma anche alle categorie dei soggetti. Se a livello nazionale questo è un diritto, realmente applicabile, non si può dire lo steso a livello internazionale. A livello pratico possono gli organismi internazionali garantire ai soggetti interessati un tale diritto di partecipazione?E' possibile che una procedura a livello internazionale avanzi se si sottoponesse all'istituto della consultazione?

Perchè, per illustrare, si capisce che in una decisione a livello nazionale, un'imprenditore oppure certi gruppi di interesse possono avere gli strumenti sufficienti per informarsi preliminarmente su una norma con impatto ambientale ma che potrebbe anche toccare i loro interessi.Loro hanno la possibilità di esporre le loro pretese attraverso le correzioni e le modifiche di legge, cioè esercitano il diritto di consultazione. Ma può questo accadere a livello globale, cioè quando la decisione viene presa da un organismo internazionale? 
Gli esempi sono tanti e questo normalmente ti porta all'idea di formulare una teoria del nuovo ordine giuridico e subito dopo un processo di positivizzazione della globalizazzione giuridica. Un processo molto complicato per diversi motivi: innanzitutto perche è obbligatorio. I processi globalizzanti sono una strada senza ritorno, cosa che obbliga il diritto di riconcepirsi entro questa tendenza.Secondo, perchè la regolarizzazione della globalizzazione giuridica è, per definizione, universalizzante e tende a rinforzare l'unita giuridica mondiale. Questo si contrappone alla molteplicita dei sistemi giuridici locali. Terzo, alla globalizzazione giuridica manca l'autorita legittimante. Questa autorità non puo essere un organizzazione internazionale come si intende oggi, non puo essere un'alleanza tra Stati e tanto meno un'unico Stato, indipendentemente dalla sua potenza. Lo dice Gunter Teubner: "...il diritto globale che si sta evidenziando oggi, è un sistema in sè, che non va comparato con i standard dei sistemi giuridici nazionali o transnazionali".

Comunque, questo tipo di diritto può essere di successo soltanto se viene concepito nell'ottica del pluralismo giuridico. II che vuol dire, un processo di armonizzazione e riconciliazione dei diversi sistemi giuridici (e non soltanto quelli statali). Questo, prima di tutto, significa riconcepire il diritto stesso, in termini pluralistici. Questo si può fare, come saggiamente riassume Rodolfo Sacco "...superando tre ostacoli : quello politico-territoriale, che significa gli ostacoli tra gli Stati; quello cognitivo, che significa gli ostacoli tra gli operatori del diritto; quello disciplinare, che significa gli ostacoli tra il diritto e le altre discipline". Soltanto se concepito in un'ottica pluralistica il diritto può riuscire ad adattarsi alle dinamiche della globalizzazione. Anzi, riconcepito così, può essere il più affidabile portavoce di queste dinamiche.

\section{II bio-giuridico}

II fatto che i giuristi si sono impegnati in ritardo nel dibattito sulla questione bioetica, era legato ad un'incertezza relativa al loro status in questo dibattito. In linea generale avevano ragione: il dibattito sopramenzionato era trasformato fino a quel tempo, in un dominio tra la scienza (in particolare la biologia) e la morale. L'entrata nel dibatito della scienza del diritto coincide più o meno con la dichiarazione dell'oncologo americano Van Potter nella sua opera "Bioethics. Bridge to the future", secondo il quale "il sapere tradizionale, sia quello scientifico, sia quello'etico non sono capaci di affrontare le nuove problematiche provocate dalla scienza biologica". (Bioethics. Bridge to the future, Englewood Cliffs, New Jersey, 1971).

Strettamente collegato con la sopradetta causa, un'altro motivo che giustificava la difficoltà del diritto di intervenire in questo dibatito, era legato con la natura delle domande che pone in essere la bioetica perchè, in prima vista sono domande che si rivolgono direttamente alla morale.

Devono esistere dei limiti per intervenire nel corpo umano? L'aborto è parzialmente, eugenismo? L'embrione umano va considerato un'entità senza identita oppure persona umana? L'eutanasia, essenzialmente, è un permesso di uccidere? Nel caso della fecondazione in vitro chi è la madre anagrafica del nascituro,l a madre biologica 0 quella surrogato? Queste sono alcune delle infinite domande che pone la bioetica.

Non c'è dubio che il diritto non può normativizzare la coscienza ma è altretanto vero che la vita umana in molti dei suoi momenti essenziali esce fuori dalla sfera privata per realizzarsi in quella pubblica. Non è il caso degli aspetti sociali come l'istruzione, l'occupazione, il matrimonio, il benessere ecc. No! Si parla degli aspetti biologici della vita, nascita e morte, in tutte le variabili che esse celano dentro di sé. E dentro la sfera pubblica il dirtto non può stare mai in silenzio. È per antonomasia la sua dimensione. II diritto è l'unico che gode il diritto di "giuridificare" il corpo: per disciplinare normativamente il rapporto tra la fisicità dell'individuo e la sua identità; per spostare nella sfera pubblica aspetti che fino a poco tempo fa erano chiusi entro la sua limitata sfera privata; per stabilizzare il "regolamento" della "governazione del corpo". (Trattato di biodiritto, Rodotà .S., Zatti.P., Giuffrè, Milano).

Le domande che pone la bioetica sono molto complesse e pongono il diritto in una posizione in cui deve scoprire nuove categorie con le quali non ci siamo abituati a ragionare. Sottintende che l'impostazione classica sulla bioetica in una chiave esclusivamente morale è decisamente insufficiente. Certamente questo non dà diritto alle posizioni che portano verso una relativizzazione morale entro il contesto sociale, nel trattamento dei casi di bioetica. Questa sarebbe un'alternativa irrazionale. Ė molto importante quello che sottolinea Hare quando si appella al bisogno di identificare regole della verità immutabili, indipendentemente dai contesti sociali:" Possiamo raggiungere l'obbietività nelle nostre formulazioni morali se il lessico e la logica al quale essi appartengono costringono tutti quelli che ragionano in maniera razionale di giungere alle stesse opinioni morali, anche se partono da diversi punti di vista. (Hare.R, Obiective prescriptions, in "Philosophical Issue", volume 4, 1993). Ma il ragionamento di Hare viene completato quando accetta il diritto di scegliere un'etica la quale: "si deve basare sull'accetazzione razionale della verità da chiunque detenga la conoscenza dei fatti non morali" (Hare.R,Scegliere un'etica,Il Mulino,pg.176). 
Ma dopotutto, questo è il comportamento morale riguardante i problemi della bioetica. Comportamento corretto sicuramente ma come abbiamo detto insufficiente. Allora come si deve comportare il diritto riguardo a queste problematiche?

Prima di tutto partendo dal prerequisito che, l'implicazione nei casi di bioetica non ha a che fare con la costruzione dell'immagine dell'individuo moderno ma con la difesa della sua identità. Ci basta portare due esempi per provare questo: il feto, o l'embrione non gode lo status di persona secondo il codice civile ma il diritto lo riconosce come centro gravitazionale degli interessi che meritano tutela giuridica visto che si trova là il punto di partenza biologico e giuridico della persona adulta ; anche per il malato terminale vale esattamente la disposzione costituzionale.

"La vita viene protetta dalla legge" (Costituzione d'Albania, art.21) e solo il cosiddetto testamento biologico (categoria della biogiuridica) sarebbe lo strumento giuridico adatto per rispettare la dignità della vita dell'individuo.

Però allo stesso tempo l'intervento giuridico nei casi di bioetica deve fare i conti anche con la struttura morale degli suoi interpreti. Accettando che, l'interpretazione non è un momento passivo applicativo ma una vera attività sul diritto, è impossibile che l'interprete non venga toccato in questo processo decisionale dai suoi sentimenti soggettivi e morali. Questo sarebbe un rischio imminente nei casi di un quadro normativo completato ma anche di più nei casi di quadri normativi con delle lacune nel campo di bioetica. Però allo stesso tempo l'intervento del diritto nei casi di bioetica è un operazione che rivalorizza lo stesso diritto. Esso cerca di spiegare la validità e l'efficacia delle categorie giuridiche alla luce dei cambiamenti sceintifici, sociali-culturali e quelli morali. Perchè, "la legge come forma di regolamentazione dei casi di bioetica, rischia da una parte di essere sempre in ritardo fisiologico, superata velocemente dai cambiamenti della società contemporanea e dalle continue scoperte scientifiche, e dall'altra imporre valori che non sono comuni e che si affermano solo attraverso l'adozione di procedure maggioritarie"(Casonato. C., Introduzione al biodiritto, Giapiccheli, Torino, 2009).

Il diritto deve fare i conti anche nei casi in cui cerca positivizzare fenomeni di natura bioetica ed anche la struttura morale della società in un determinato periodo storico. Normalmente nascono le domande: Hanno la priorità i diritti dell'individuo oppure i diritti di gruppi che sono minoranza in società, oppure la priorità assoluta spetta alla maggioranza dell società la quale non è pronta moralmente di accettare i suoi diritti? Deve il diritto essere semplicemente una prerogativa dell' individuo però senza dare a quella prerogativa quello speciale status giuridico che le permeterebbe la difesa da parte della legge?

Le risposte sonon difficili per il diritto, per la biogirudica più precisamente,. Si deve ammettere che nei casi di bioetica il diritto si trova di fronte agli "eccessi" (non nel senso negativo) delle vita. Per disciplinare in maniera più corretta questi "eccessi" si deve distanziare dalla vita e vedere il tutto freddamente ed in equilibrio. Ma si deve distanziare anche dal concetto di diritto come comando autoritario. La biogirudica è interdisciplinare che vuol dire che diritto per normatizzare la bioetica ha bisgno di dialogare sotto il principio della toleranza anche con la morale, anche con la politica, anche con la scienza ed anche con la religione. Sono tutte, così come il diritto, esperienze coesistenziali. Ma diversamente dal diritto, sono strutture chiuse perchè a differenza del diritto garantiscono la loro esistenza nell' omogeneità dentro al gruppo. Ma il diritto è una struttura aperta senza confini politici, religiosi, morali, ideologici oppure scientifici. Proprio questa sua dote meravigliosa forma, paradossalmente, anche la sua reale difficoltà di regolare "il nuovo modo dell'esistenza di tutto il ciclo della vita, la nascita, l'esistenza, la morte fino a "prima" e "dopo" di essa".

\section{Bibliografia}

Bioethics.Bridge to the future,Englewood Cliffs, New Jersey,1971.

Faralli Carla,Filosofia del diritto contemporanea,Laterza,2002

Cassese.S,Universalita del diritto, Napoli,Ed. Scientifica,pg.7,2005.

Casonato.C.,Introduzione al biodiritto,Giapiccheli,Torino,2009

Fundamental legal conceptions as applied in judicial reasoning,1917,f.71

Gurvitch.G,L'idèè du droit social,Paris,1932,pg,119.

Gurvitch.G, La declaration des droits sociaux,Edizioni di comunita,Milano,1949.

Hare.R,Obiective prescriptions,në "Philosophical Issue",volume 4,1993

Hare.R,Scegliere un'etica,II Mulino,f.176.

Costituzione d'Albania,art. 21

Lon Fuller, The morality of law,University of Yale,1969.

Sperber.D, La sapienza degli antropologi,Milano,pg.57-58.

Viola.F,Concorrenza degli ordinamenti e il diritto come scelta, Ed.Scentifica, Napoli, pg.65, 2008.

Waldron. J,Foreign law and the modern ius gentium, Harward Law Review,pg.130,2005 